\title{
Production of Polyunsaturated Fatty Acids by Genetic Engineering of Yeast
}

\author{
Yasunari MICHINAKA ${ }^{* 1}$, Tsunehiro AKI ${ }^{* 1}$, Katsuya INAGAKI ${ }^{* 1}$, Hirofumi HIGASHIMOTo*1, \\ Yayoi SHIMADA ${ }^{* 1}$, Toshiaki NAKAJIMA ${ }^{* 2}$, Toshitsugu ShIMAUCHI ${ }^{* 2}$, \\ Kazuhisa $\mathrm{ONO}^{* 1}$ and Osamu SUZUKI ${ }^{* 1 *}$ \\ *1 Department of Molecular Biotechnology, Graduate School of Advanced Sciences of Matter, Hiroshima University \\ (Higashi-Hiroshima, JAPAN) \\ *2 Technical Center, Idemitsu Technofine Co. Ltd., \\ (Tokyo, JAPAN)
}

Edited by T. Nakahara, AIST, Tsukuba, and accepted January 20, 2001 (received for review October 23, 2000)

\begin{abstract}
In order to study the production system of polyunsaturated fatty acids (PUFA), we genetically engineered a baker's yeast that did not originally produce PUFA. We produced $\gamma$ linolenic acid (GLA) by constructing a heterologous system to express $\Delta 6$ fatty acid desaturase (D6d) gene from rat liver into yeast, Saccharomyces cerevisiae. The expression of this gene in the presence of linoleic acid (LA) formed a significant amount of GLA in the host cells. In order to effect the largest expression of the conversion rate from LA to GLA, we set up a dual promoter system, where genes coding for the D6d and an electron donor protein, cytochrome $b_{5}$, were coordinately expressed. The desaturation index (GLA/LA) increased significantly by high level expression of cytochrome $b_{5}$, indicating that this protein is a limiting factor in regular yeast. In addition, we examined a condition for the extra-cellular production of GLA by a fatty acid secretion yeast mutant. By expressing the D6d gene under the control of GAP-DH promoter in the presence of LA, $178 \mathrm{mg} / \mathrm{L}$ of GLA was secreted in the medium in $144 \mathrm{~h}$.

J. Oleo Sci. 50, 359-365 (2001).

Key words : genetic engineering, polyunsaturated fatty acids, $\gamma$-linolenic acid, $\Delta 6$ fatty acid desaturase, Saccharomyces cerevisiae, cytochrome $b_{5}$, fatty acid secretion mutant
\end{abstract}

\section{Introduction}

$\gamma$-Linolenic acid (GLA, C18:3 $\Delta 6,9,12$ ) is now commercialized as a fatty acid component in plant seed oils from evening primrose and borage as well as in microbial production oils, termed single cell oils (SCO). Fifteen years ago, our research group in the National Chemical Laboratory for Industry cooperated with private companies, such as Idemitsu Co. Inc. and Shiseido Co., as the first in the world (1-2) to industrialize production technology for SCO containing GLA (GLASCO). We used a fungus belonging to the genus Mortierella as a source of SCO. Commercial products using GLASCO-based material, such as health foods and cosmetics, had by then come into the market (3). Despite being a small market, it was viewed as a leading example for production of other kinds of SCO, especially oils containing polyunsatu- rated fatty acids (PUFA). Subsequently, many studies on biological functions and utilization have been pursued for commercial SCOs that contain arachidonic acid (ARA, C20:4 $\Delta 5,8,11,14$ ), eicosapentaenoic acid (EPA, C20:5 $\Delta 5,8,11,14,17)$ and/or docosahexaenoic acid (DHA, C22:6 $\Delta 4,7,10,13,16,19)$ (4).

The biosynthesis of GLA in eukaryotes is accomplished by a desaturation of linoleic acid (LA, C18:2 $\Delta 9,12)(5)$. This reaction is catalyzed by $\Delta 6$ fatty acid desaturase (D6d) through an insertion of a double bond between carbons 6 and 7 (the numbers counted from carboxyl-end) of the fatty acid substrate. D6d distributes in a wide range of organisms including animals, GLA-producing microorganisms such as Zygomycetes and Oomycetes, and also some species in plants such as evening primrose and borage. Recently, genes coding for D6d were isolated from borage (Borago officinalis) (6), nematode worm 
(Caenorhabditis elegans) (7), fungus (Mortierella alpina) (8), human (9), mouse and rat (10). In this paper, the authors describe the cDNA cloning and structural characteristics of rat liver D6d and the established expression system in yeast, $S$. cerevisiae, to hyper-produce GLA. Furthermore, we discuss the use of yeast mutant for the secretion production of GLA.

\section{Molecular Cloning of Rat $\Delta 6$ Fatty Acid Desaturase}

By taking advantage of the DNA sequence database (DNA Data Bank of Japan), we tried "in silico" cDNA cloning of the fatty acid desaturase from rodents, using a conserved motif of borage and nematode desaturases as a query. The retrieved information of the expressed sequence tag (EST) indicated some rodent cDNAs to be homologous to the query and was used to clone the corresponding DNA fragments by polymerase-chain reaction (PCR) method. The resultant PCR products were subsequently used to screen a rat liver cDNA library, yielding a 1573 base pairs (bp) clone. The comparisons of the deduced amino acid sequence of the clone with those of D6ds from $C$. elegans and B. officinalis showed homology scores of $27.9 \%$ and $26.4 \%$, respectively (Fig. 1). Although the overall homology is not remarkable, we noted that a typical hemebinding motif, HPGG, and three histidine clusters, $\mathrm{HX}_{2}$ or ${ }_{3} \mathrm{HH}, \mathrm{HX}_{3}$ or ${ }_{4} \mathrm{H}$ or $\mathrm{QX}_{2} \mathrm{HH}$, that conserved highly within the desaturase family were found in our clone as well as others.

For functional analysis of the rat desaturase-like gene, a plasmid expressing the largest coding region (1332 bp, 444 amino acids) was constructed on an expression vector pYES2 for the baker's yeast. The gene was under the control of GAL1 promoter and therefore inducible by the presense of galactose. After obtaining yeast transformant carrying the plasmid, cells were cultivated, supplemented by the addition of substrate fatty acid, and induced. An aliquot of cells was harvested for analysis of intercellular fatty acid composition by gas-liquid chromatography (GC). Figure 2 shows the typical chromatograms of fatty acid methyl esters from the transgenic yeast. When LA was supplied as a substrate to the growing culture of the transformed yeast, a novel peak corresponding to GLA appeared in the chromatogram, and subsequent analysis on GC-mass spectrometry supported the identity of the peak as a methyl ester form of GLA (data not shown). Similarly, the addition of $\alpha$-linolenic acid (ALA, C18:3 $\Delta 9,12,15$ ) resulted in the accumulation of its $\Delta 6$ desaturated product, stearidonic acid $(\mathrm{C} 18: 4 \Delta 6,9,12,15)$. On the other hand,

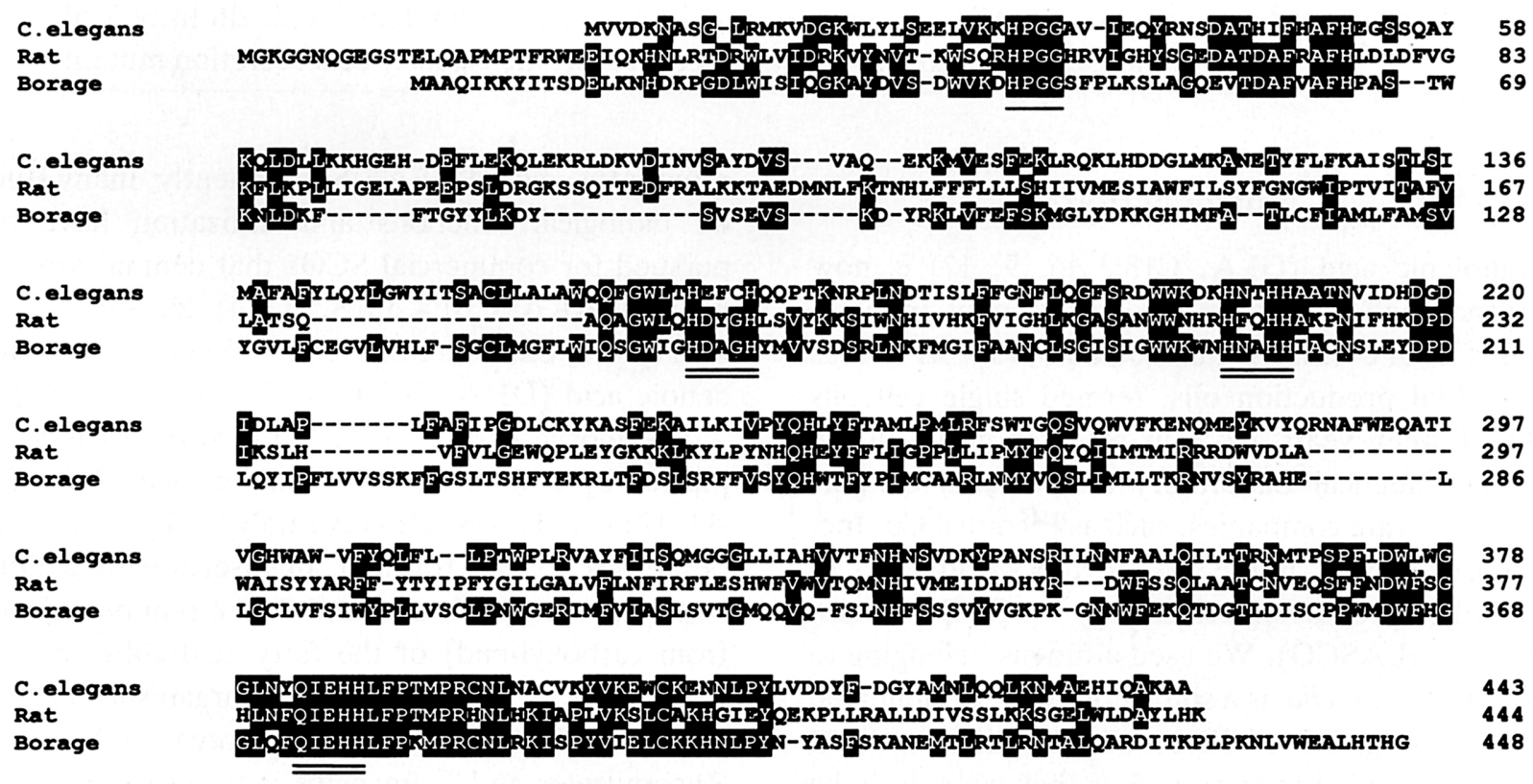

Fig. 1 Comparisons of the Deduced Amino Acid Sequence of D6d from Rat Liver with Those from B. officinalis (GenBank accession number U79010) and C. elegans (AF031477). Identical residues are shaded, and the conserved heme-binding motif and three histidine clusters are single- and double-underlined, respectively. 


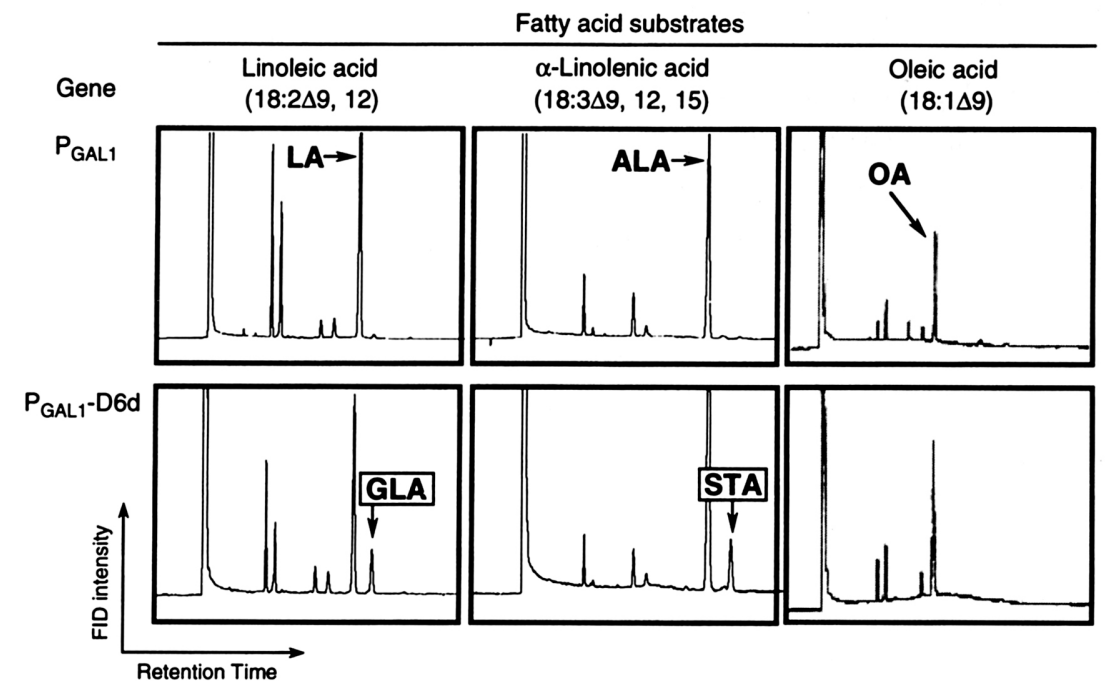

Fig. 2 Expression of the Rat D6d in Yeast in the Presense of $\Delta 9$ Fatty Acids. Plasmid carrying a GAL1 promoter only or that followed by the rat D6d gene were introduced into the yeast and expressed in the presense of indicated fatty acid substrates.

dihomo- $\gamma$-linolenic acid $(\mathrm{C} 20: 3 \Delta 8,11,14)$, which was recognized as a substrate for $\Delta 5$ desaturase, failed to be a substrate in our system. These results, therefore, indicate the protein encoded by the cloned cDNA was D6d.

Moreover, in the rat D6d cDNA, another translation start site (ATG codon) was found in-frame at 51 nucleotides downstream of the ATG at most upstream (Fig. 1). Since we could not determine which site was the proper one, the 5'-truncated version of D6d gene (1281 bp, 427 amino acids) was also tested whether it functionally expressed in yeast. As a result, we could not see any significant differences between those two series of expression analyses in terms of substrate specificity, conversion rate, and nature of the products (data not shown). This suggests that the first 17 amino acids in the larger fragment are not required to function, at least in yeast. Determination of the amino-terminal end of the D6d protein in rat liver is under the way.

\section{Substrate Specificity of Rat $\Delta 6$ Fatty Acid Desaturase}

In the established pathway of PUFA biosynthesis in mammalian cells, there are at least five steps in which D6d is involved; three of them are for C18 fatty acid substrates and two others for $\mathrm{C} 24(5,11)$. To date, no clear conclusion has been made whether multiple forms of D6d exist. However, an in vivo metabolic study by Christiansen et al. (12) indicated that liver microsomes might contain separate enzymes for the desaturation of LA and ALA, which responded differently to dietary fats. More recently, Rodriguez $e t$ al. (13) strongly suggested the existence of two distinct D6ds by the fact that N-ethyl maleimide (NEM) inhibited in vitro the microsomal $\Delta 6$ desaturation of LA but activated that of ALA. Nevertheless, it is obvious in our results that the rat D6d expressed in yeast exhibits no substrate selectivity toward LA and ALA (Fig. 2). Those fatty acid substrates were $\Delta 6$ desaturated at the equivalent level of conversion efficiency. It may be interesting to examine the effect of NEM on our system.

When essential fatty acids such as LA and ALA are not sufficiently supplied, a pathway for biosynthesis of mead acid $(C 20: 3 \Delta 5,8,11)$ is activated in mammalian cells, thought to contribute to maintenance of membrane fluidity (14). In this $\mathrm{n}-9$ pathway, a precursor, oleic acid (OA, C18:1 $\Delta 9$ ), should be desaturated first at $\Delta 6$ position, and therefore D6d could participate in this reaction as well. However, in our yeast expression system of D6d, either endogenous or supplemented OA was not converted to the expected $\Delta 6$ fatty acid (Fig. 2). Accordingly, there could exist another type of D6d which preferentially acts on OA.

Sprecher et al. (15) proposed a novel pathway for 
DHA biosynthesis, which includes a $\Delta 6$ desaturation step of tetracosapentaenoic acid (C24:5 $\Delta 9,12,15,18$, 21) followed by peroxisomal $\beta$-oxidation. A similar pathway was found in the biosynthesis of $n-6$ docosapentaenoic acid (C22:5 $\Delta 4,7,10,13,16)$. A series of in vitro studies by these authors suggested that a single D6d might participate in the $\Delta 6$ desaturation of both $\mathrm{C} 18$ and $\mathrm{C} 24$ fatty acids (16). This may prove conclusive if C24 PUFAs become available to test as substrate fatty acids for the D6d expression system in yeast. Nevertheless, it should be noted that a main pathway of DHA biosynthesis might be in the mitochondria, which includes a $\Delta 4$ desaturation step, excluding the involvement of D6d in the endoplasmic reticulum (17).

\section{The Role of Cytochrome $b_{5}$ in the $\Delta 6$ Fatty Acid Desaturation System}

Membrane-bound desaturases that have been cloned from a wide range of organisms have a structural similarity: two hydrophobic domains and three histidine-rich clusters (6-10). Some His residues in the latter is thought to interact with a non-heme iron, which transfers an electron to the fatty acid substrate to be desaturated. In mammalian cells, such an electron is served by a donor protein, cytochrome $b_{5}$, and the redox status of this protein is modulated by NADH-dependent cytochrome $b_{5}$ reductase (14). The postulated scheme of the electron transfer system in rat $\Delta 9$ desaturation complex is illustrated in Fig. 3A. On the other hand, the $\Delta 9$ desaturation system in yeast, does not require the microsomal cytochrome $b_{5}$
A

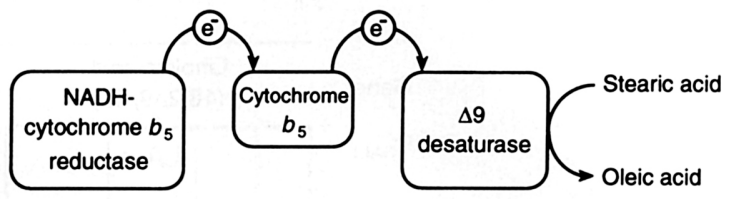

B

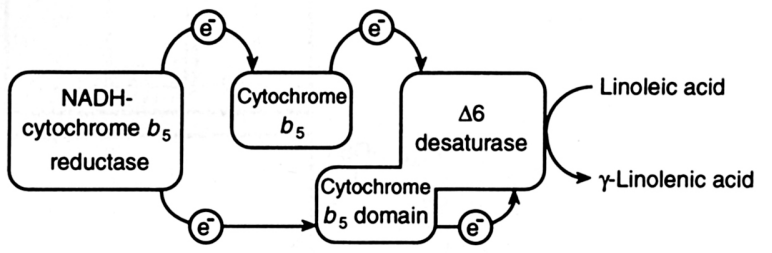

Fig. 3 Diagramatic Representation of the Postulated Electron Transfer System on the $\Delta 9$ (A) and $\Delta 6$ (B) Desaturation Complexes.

(18). It is considered this to be due to a functional cytochrome $b_{5}$ module that exists in the carboxylterminal region of yeast $\Delta 9$ desaturase, OLE1, whereas the rat counterpart lacks this domain (Fig. 4). Interestingly, the cytochrome $b_{5}$-like domain has also been found in the amino-terminal region of the so-called "front-end" type desaturases such as D6d from fungi and mammals (19). This led us to suppose that, in mammals, the microsomal cytochrome $b_{5}$ is essential for $\Delta 9$ desaturation but not for $\Delta 6$ desaturation.

To test this possibility, we used a system similar to that above, where the rat D6d carrying the cytochrome $b_{5}$ domain at the amino-terminal region was expressed in a cytochrome $b_{5}$-deficient yeast. The rat $\Delta 9$ desaturase did not function in this mutant (18). In contrast, we observed (Table 1) that an

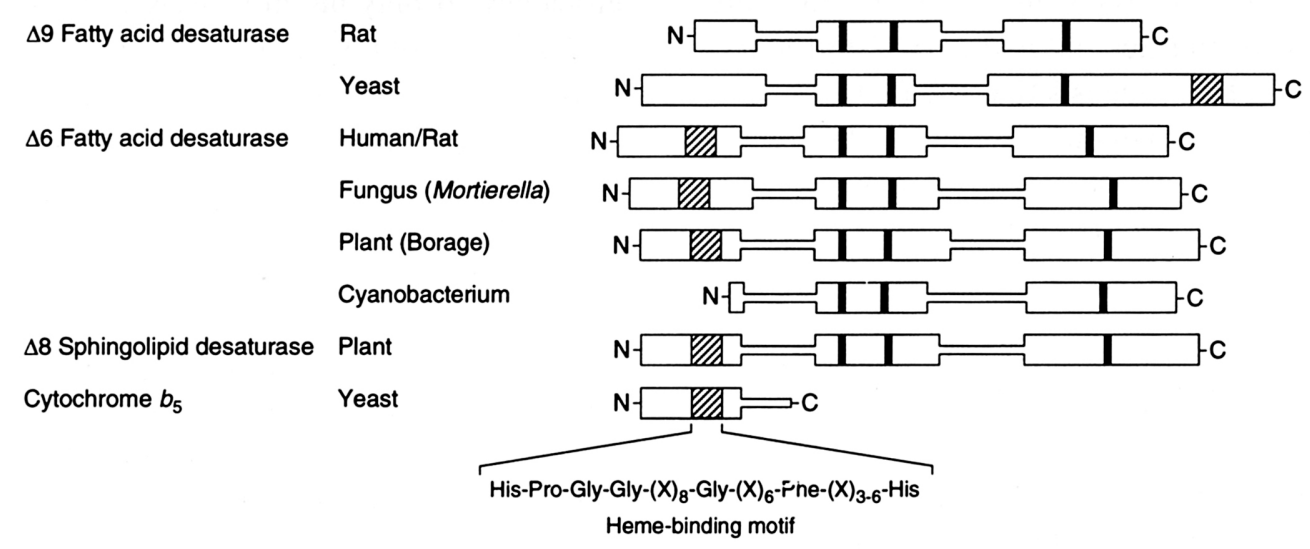

Fig. 4 Structural Comparisons of Fatty Acid Desaturases and Related Enzymes. The hemebinding regions and histidine clusters are indicated by hatched and shaded boxes, respectively. 
Table 1 Expression of Rat D6d Gene in Wild-type (DTY-10a) and Cytochrome $b_{5}$-deficient (AMY$1 \alpha)$ Yeasts.

\begin{tabular}{llccl}
\hline S. cerevisiae strain & Plasmid & \multicolumn{2}{c}{ Relative content $(\%)$} & \multirow{2}{*}{ Ratio of GLA to LA } \\
\cline { 3 - 4 } & & LA & GLA & \\
\hline DTY-10a & pYES2 & 100 & 0 & 0 \\
& pYES2/D6d * & $74.4 \pm 4.6$ & $25.6 \pm 3.4$ & $0.34 \pm 0.06$ \\
AMY-1 $\alpha^{*}$ & pYES2 & 100 & 0 & 0 \\
& pYES2/D6d * & $87.9 \pm 6.2$ & $12.1 \pm 4.9$ & $0.14 \pm 0.05^{\text {a }}$ \\
\hline
\end{tabular}

* Rat D9 desaturase lacking cytochrome $b_{5}$-domain was not functionally expressed in cytochrome $b_{5^{-}}$ null situation (18).

** Equivalent level of D6d protein was observed by an immunoblotting analysis of total cell extracts from D6d-expressing yeast transformants.

a Significantly different from the case of wild type host $(\mathrm{p}<0.05)$.

exogeneously-added LA was converted to GLA in both the wild type and the mutant, suggesting that the microsomal (host-derived) cytochrome $b_{5}$ was not neccessary for the D6d to function. However, when the substrate LA was added at the saturating amount, we observed with interest that the $\Delta 6$ desaturation index (the ratio of GLA to LA) in the mutant was apparently lower than that in the wild type. We then postulated an involvement of the microsomal cytochrome $b_{5}$ in the desaturation reaction. To address this, we constructed a plasmid carrying both the D6d and cytochrome $b_{5}$ genes, and used it to co-express those two proteins simultaneously in yeast. As a result, the hyper-expression of cytochrome $b_{5}$ as well as D6d elevated the $\Delta 6$ desaturation index about 2fold in wild type cells and more than 3 -fold in the cytochrome $b_{5}$-deficient mutant (Fig. 5). These data imply that the microsomal cytochrome $b_{5}$ takes part in the desaturation reaction and is necessary to exhibit full activity of the $\Delta 6$ desaturation complex. It may be important to regulate the expression of cytochrome $b_{5}$ for mass production of GLA in microorganisms and plants.

Moreover, we evaluated an essential role of $\mathrm{His}^{53}$ of the rat D6d by a site-directed mutagenesis. The $\mathrm{His}^{53}$ resides in the heme-binding motif, HPGG, of cytochrome $b_{5}$ domain. A mutant D6d protein in which $\mathrm{His}^{53}$ was substituted by an Ala was successfully expressed in yeast and recovered in a membrane fraction. However, yeast cells expressing this mutant D6d did not show an ability to convert LA to GLA (Fig. 6). Sayanova et al. (20) reported essentially the same result on the borage D6d by mutation of the corresponding site. These results may indicate that the cytochrome $b_{5}$ mutant fused with D6d lost its function,
A

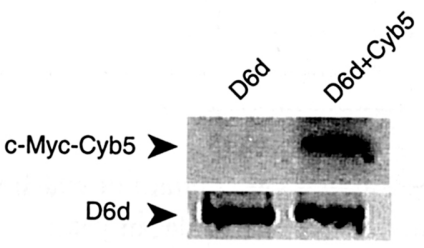

B

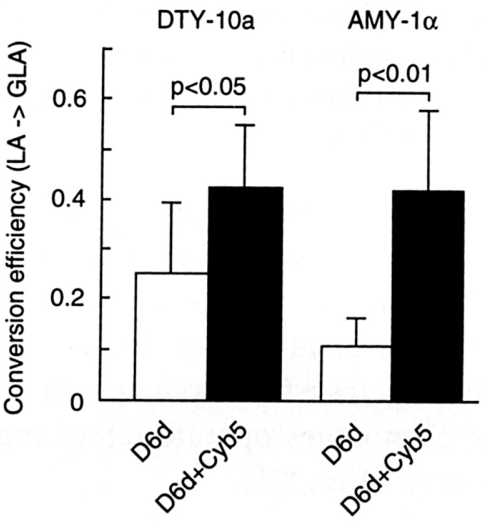

Fig. 5 Co-expression of the Rat D6d with Cytochrome $b_{5}$ in Yeast. The rat D6d and cytochrome $b_{5}$ genes are inserted to an yeast expression vector pESC-URA (Stratagene), which are fused with FLAG and c-Myc peptide tags and are under the controls of GAL10 and GAL1 promoters, respectively. (A) Immunoblotting analyses of yeast cell extract from AMY- $1 \alpha$ carrying only D6d gene or both D6d and cytochrome $b_{5}$ genes, using anti-c-Myc peptide antibody (upper panel) or anti-rat D6d antibody (lower panel). (B) Fatty acid analysis of yeast cells expressing D6d and cytochrome $b_{5}$ in the presense of LA. Results show conversion efficiency which are calculated as GLA per LA. Cyb5, cytochrome $b_{5}$. 
A

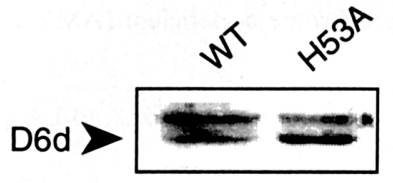

B
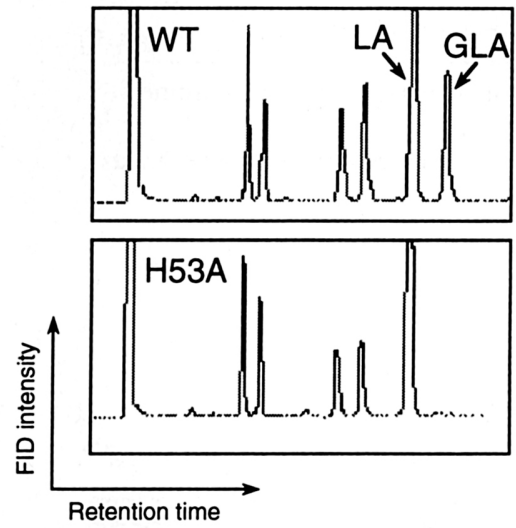

Fig. 6 Expression of a D6d mutant of which the $\mathrm{His}^{53}$ residue was substituted by an Ala, in yeast . (A) Immunoblotting analysis of yeast cell extracts confirming the expression of wild type (WT) and mutant D6d (H53A) proteins using anti-rat D6d antibody. (B) GC profiles of cellular total fatty acid from the yeast transformants that expressed wild type and mutated D6d genes in the presense of LA.

due to an inability to supply heme-binding sites. Taken together, we assume that both microsomal and fused cytochrome $b_{5} \mathrm{~s}$ are essential for maximum activity of the $\Delta 6$ desaturation. Hence, we propose a novel electron transfer system, in which two cytochrome $b_{5}$ modules operate either independently or coordinately (Fig. 3B).

\section{Secretion Production of GLA by Genetic Engineering of Yeast}

We previously isolated a yeast mutant YTS51 and its backcross strain C7-4b that secrete the free form of fatty acids (21). Here, we studied secretion production of GLA through the introduction and expression of rat D6d gene to those mutants. When the mutants and wild type cells were cultivated for up to $130 \mathrm{~h}$, the C7$4 \mathrm{~b}$ showed the highest amount $(0.34 \mathrm{~g} / \mathrm{L})$ of extracellular fatty acids although its cell growth and the amount of intracellular fatty acids were not superior to the wild type (Fig. 7). The extracellular fatty acids were identified as a free form by thin-layer chromatography (data not shown).

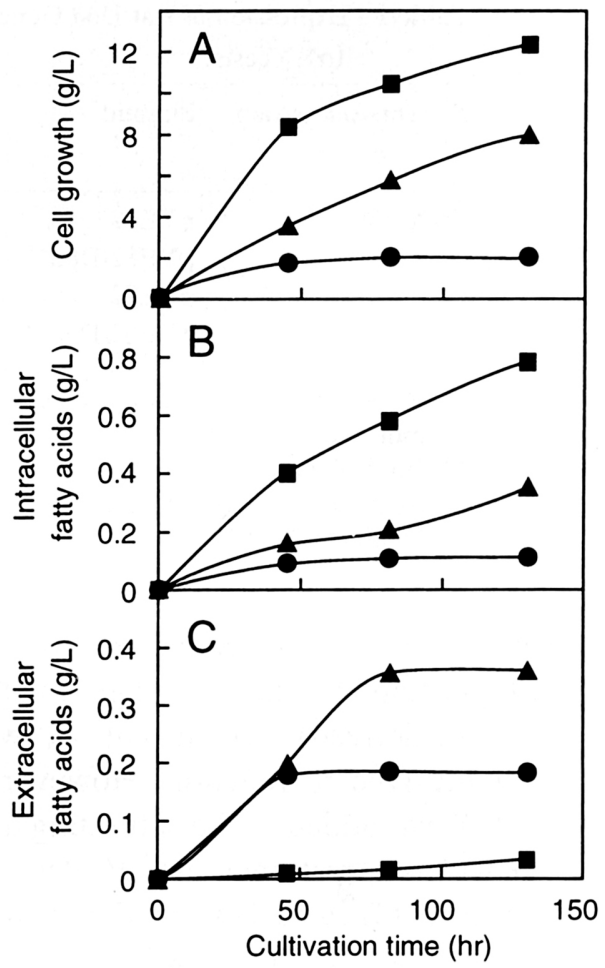

Fig. 7 Secretion production of fatty acids by $S$. cerevisiae mutants. Yeast cells were cultivated in complete medium (2\% glucose, $5 \%$ glycerol, $2 \%$ peptone, $1 \%$ yeast extract, $0.5 \%$ Brij-58). Cell growth (A) and intra(B) and extra-cellular (C) fatty acids were measured during the cultivation. Squares, C1-6b (wild type); circles, YTS-51 (fatty acid secretion mutant) ; triangles, C7-4b (a backcross of YTS-51 and C1-6b).

We constructed a plasmid carrying the rat D6d gene, whose expression was controlled by a glycelaldehye-3-phosphate dehydrogenese (GAP$\mathrm{DH})$ promoter, then introduced it into the fatty acid secretion mutants. By cultivations of the transformants, YTS51/D6d and C7-4b/D6d, in the presense of a substrate LA for $144 \mathrm{~h}$ together with a glucose supplementation, $178 \mathrm{mg} / \mathrm{L}$ and $117 \mathrm{mg} / \mathrm{L}$ of extracellular GLA was observed, respectively (Fig. 8). Although the productivity of GLA is not yet at a satisfying level, we have shown the possibility of secretion production of GLA by using the transformation of oil secretion yeast mutant.

Since we developed the first industrial application of GLASCO production, studies including gene cloning have examined D6d as a rate-limiting factor for improving productivity. In this paper we introduced a line of basic studies for a new type of GLA production technology using a combination of heterologous D6d 


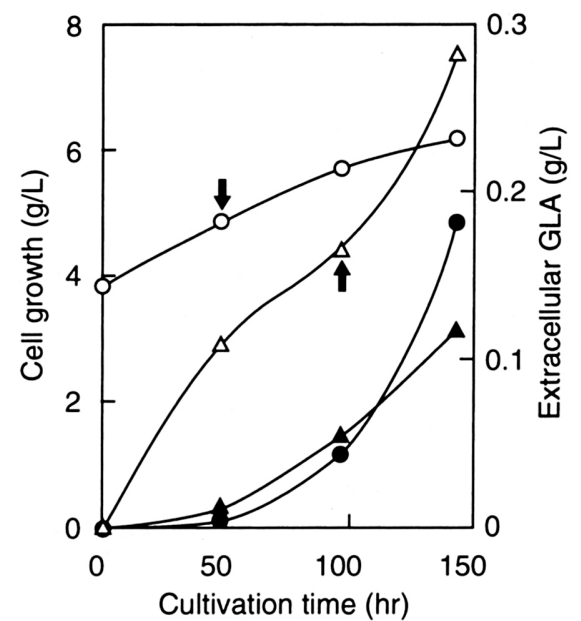

Fig. 8 Secretion production of GLA by expression of the rat D6d in $S$. cerevisiae mutants. Fatty acid secretion mutants, YTS-51 (circles) and C74b (triangles), were transformed with plasmid carrying the rat D6d gene. Yeast cells were cultivated in a medium ( $2 \%$ glucose, $5 \%$ glycerol, $0.67 \%$ yeast nitrogen base without amino acids, $1 \%$ Tergitol NP-40) supplemented with $1 \mathrm{~g} / \mathrm{L}$ of linoleic acid. Glucose was added to $2 \%$ at the time indicated by solid arrows. Cell growth (open symbols) and extracellular GLA (closed symbols) were measured during the cultivation.

expression and oil secretion yeast mutant. In particular, the host cells' characteristic secretion of free form fatty acids will eliminate the cell-crushing step in oil processing and is easy to use for synthesis of the structured lipids. Furthermore, it should be interesting to introduce other types of fatty acid modifying enzymes such as $\Delta 12$ and $\Delta 5$ desaturases and elongase to produce PUFAs by using yeast as a bioconversion apparatus.

\section{References}

1. Suzuki, O. (1988) Proceeding of World Conference on Biotechnology for Fats and Oils Industry, Hamburg, pp.110-116.

2. Suzuki, O., Yokochi, T. \& Nakahara, T. (1996)
Proceedings of the 1996 PORIM Industrial Palm Oil Congress, Malaysia, pp.103-113.

3. Yokochi, T., Suzuki, O. \& Nakasato, T. (1988) Jpn. Pat., 1467116.

4. Mukherjee, K.D. (1999) INFORM, 10, 308-313.

5. Horrobin, D.F. (1992) Prog. Lipid Res., 31, 163-194.

6. Sayanova, O., Smith, M.A., Lapinskas, P., Stobart, A.K., Dobson, G., Christie, W.W., Shewry, P.R. \& Napier, J.A., (1997) Proc. Natl. Acad. Sci. USA, 94, 4211-4216.

7. Napier, J.A., Hey, S. J., Lacey, D.J. \& Shewry, P.R. (1998) Biochem. J., 330, 611-614.

8. Michaelson, L.V., Lazarus, C.M., Griffiths, G., Napier, J.A. \& Stobart, A.K. (1998) J. Biol. Chem., 273, 19055-19059.

9. Cho, H.P., Nakamura, M.T. \& Clarke, S.D. (1999) J. Biol. Chem., 274, 471-477.

10. Aki, T., Shimada, Y., Inagaki, K., Higashimoto, H., Kawamoto, S., Shigeta, S., Ono, K. \& Suzuki, O. (1999) Biochem. Biophys. Res. Commun., 255, 575579.

11. Voss, A., Reinhart, M., Sankarappa, S. \& Sprecher, H. (1991) J. Biol. Chem., 266, 19995-20000.

12. Christiansen, E.N., Lund, J.S., Rørtveit, T. \& Rustan, A.C. (1991) Biochem. Biophys. Acta, 1082, 57-62.

13. Rodriguez, A., Sarda, P., Boulot, P., Leger, C.L. \& Descomps, B. (1999) Lipids, 34, 23-30.

14. Cook, H.W. (1996) Biochemistry of Lipids, Lipoproteins and Membranes, Elsevier Science, Amsterdam, pp.129-152.

15. Sprecher, H. (1996) Nutrition, 12, S5-S7.

16. Geiger, M., Mohammed, B.S., Sankarappa, S. \& Sprecher, H. (1993) Biochem. Biophys. Acta, 1170, 137-142.

17. Infante, J.P. \& Huszagh, V.A. (2000) FEBS Letters, 468, 1-5.

18. Mitchell, A.G. \& Martin, C.E. (1995) J. Biol. Chem., 270, 29766-29772.

19. Napier, J.A., Sayanova, O., Stobart, A.K. \& Shewry, P.R. (1997) Biochem. J., 328, 717-720.

20. Sayanova, O., Shewry, P.R. \& Napier, J.A. (1999) Plant Physiol., 121, 641-646.

21. Suzuki, O., Ono, K., Aki, T., Shimauchi, T., Nakajima, T. \& Kondo, A. (2000) Jpn. Pat., applied. 